\title{
Factores de interacción de la tecnología y la organización que inciden en la adopción de Ingeniería de Software
}

\author{
De Freitas, Vidalina \\ Viloria, Orlando \\ Álvarez, Guillermo
}

\section{Resumen}

Este artículo presenta los resultados de una investigación de campo de naturaleza descriptiva, cuyo objetivo fue evaluar las variables que influyen en la adopción de las herramientas Computer Aided Software Engineering o Ingeniería de Sottware Asistido por Computadora, CASE, en empresas venezolanas, que surgen de la interacción entre las innovaciones tecnológicas y el contexto organizacional de estas empresas, según la opinión de gerentes del nivel Gerencial alto y medio de organizaciones que han pasado por el proceso de evaluación de las CASE. La muestra objeto de estudio estuvo conformada por cuarenta (40) gerentes pertenecientes a treinta y cinco (35) empresas. EI análisis realizado ratifica que las variables que inciden en la adopción de las herramientas CASE en las organizaciones venezolanas son: la compatibilidad de la metodología con las herramientas CASE, el apoyo de la gerencia senior y, de la alta gerencia, la participación del usuario, la estrategia de implementación y el reajuste organizacional.

Palabras clave: Ingeniería de software, herramientas CASE, organizaciones, gerencia.

Recibido: 99-04-05 Aceptado: 01-04-24

* Prof. Asistente de la U.S.B. Ing. en Computación. Magister en Ing. de Sistemas (USB). Universidad Simón Bolivar. E-mail: vfreitas@ usb.ve Telf. 9063976, 016-6338062

* Prof. Agregado de la U.S.B. Lic. en Computación (UCV), Magister en Ing. de Sistemas (USB). Universidad Simón Bolivar. E-mail: oviloria usb.ve

** Prof. Agregado de la USB. Psicólogo, Lic. en Filosolía, Magister en Psicología, Magister en Educación Superior, Magister en Ing. Empresarial, Especialista en Desarrollo Organizacional, Especialista en Asesoramiento y desarrollo organizacional. Universidad Simón Bolivar.

E-mail: galvarez@usb.ve. 


\title{
Interacting Factors Between Technology and Organization Which Affect the Adoption of Software Engineering
}

\begin{abstract}
This article presents the results of descriptive research the objective of which was to evaluate the variables that affect the adoption of the Computer Aided Software Engineering (CASE) tools in Venezuelan companies. This topic evolved through the interaction between innovative technologies and the organizational context of these companies according to managers at high and middle management levels who have undergone the process of evaluating CASE. The sample population was forty (40) managers belonging to 35 companies. The analysis reaffirmed that the variables that affect adoption of the CASE tools in Venezuelan organizations are: compatibility of the methodology with CASE tools, the support of senior management and top management, the participation of the user, implementation strategy, and organizational adjustment.
\end{abstract}

Key words: Sofware engineering, CASE tools, organization, management.

\section{Introducción}

Durante los primeros años de desarrollo de las computadoras, el hardware sufrió continuos cambios, mientras el software se contemplaba simplemente como un añadido. El desarrollo del software se realizaba sin ninguna planificación, hasta que las demandas y requerimientos comenzaron a desbordarse y los costos a crecer.

La industria del software habia venido dando respuestas con una gama de herramientas anteriores a las herramientas Computer Aided Sottware Engineering ó Ingenieria de Software Asistido por Computadora, CASE, como los lenguajes de cuarta generación (4GL), pero éstas tuvieron poco impacto en la eliminación del "backlog" de aplicaciones (cantidad de software acumulado sin desarrollar) en las organizaciones, en el mantenimiento, en la documentación de los siste- mas y en la recuperación de las viejas aplicaciones que toda empresa posee.

Una definición difundida de herramientas CASE es la esbozada por Burch y Grudnitski (1992, 430), quienes al respecto señalan que, "los sistemas CASE son paquetes extensos y sofisticados con herramientas que ayudan a diseñar, desarrollar, administrar y mantener los proyectos de software". Nótese el uso de la palabra sistemas como una descripción más sofisticada de las CASE.

Las herramientas CASE son usadas en algunas de las fases de desarrollo de sistemas de información, incluyendo análisis, diseño y programación. El objetivo fundamental de la CASE es proveer un lenguaje para describir el sistema general que sea suficiente para generar todos los programas necesarios (Electronic Computer Glossary, 1996).

La CASE supone la aplicación de principios científicos a través de una me- 
todología que ayuda a producir software de alta calidad en el tiempo más corto posible. De acuerdo a Martín, (citado por Rowe 1993, 29), "para mantenerse competitivamente en el futuro, las organizaciones dependerán de la capacidad de crear rápidamente aplicaciones efectivas de computación". Las herramientas CASE proveen la velocidad requerida y si es bien implementada, sus productos serán de calidad.

Sin embargo, la CASE como cualquier otra tecnologia debe tomar en cuenta muchos factores para que ese cambio se dé de la mejor manera posible. A comienzo de los años 90 , el $70 \%$ de las organizaciones que introducian esta herramienta dejaban de usarla después de adquirida. Otras pasaron por el proceso de evaluar las herramientas, pero sentian temor de incorporarlas, ya que no poseían información respecto al impacto que éstas tendrian en cuanto a la productividad de los analistas, la actitud hacia su uso, si requerian o no cambios en su estructura organizativa, o qué criterios debian ser usados para evaluarias (Kemerer, 1992).

En Venezuela, De Freitas (1998 y 1999 ), realizo estudios sobre las variables organizacionales y tecnológicas que inciden en la adopción de las herramientas CASE. En esta investigación, se analizan los elementos que surgen de la interacción entre la tecnología y el contexto organizacional que ayudan o inhiben la adopción de las herramientas CASE, según la opinión de los gerentes de un grupo de organizaciones venezolanas de la región capital.

\section{Metodología utilizada}

Para los efectos de este estudio se realizó un análisis secundario de datos de una encuesta realizada a un grupo de gerentes de organizaciones venezolanas cuyos departamentos de desarrollo de sistemas habian pasado por el proceso de incorporar las CASE como herramienta de trabajo.

La muestra fue intencionada, no probabilistica, ya que estuvo conformada por todos los gerentes de aquellas empresas que se pudieron identificar como perteneciente a la población, ubicados en la región capital (Caracas) y de sectores tales como: industria petrolera, empresas productoras de alimentos y bebidas, sector bancario, empresas de servicios, empresas de seguros y consultoras en tecnología de la información. La encuesta fue originalmente usada para realizar el estudio de los Factores que Inciden en la Adopción de las Herramientas CASE en las Organizaciones Venezolanas (De Freitas, 1997), sin embargo, la información era suficiente para realizar estudios conexos.

En particular, se tomó el instrumento de medición considerando un banco de indicadores que estaban agrupados por factores y sivieron para generar los reactivos que conformaron la encuesta.

El análisis de resultados se estructuró en dos partes, una primera parte, referente a los Resultados Descriptivos, la cual abarca el análisis correspondiente a las características resaltantes de la muestra estudiada. La segunda parte, se relaciona con las Inferencias resultantes 
del análisis de la opinión de los gerentes respecto a las variables que surgen de la interacción entre la tecnología y el contexto organizacional que afectan la adopción de las herramientas CASE.

\section{El contexto organizacional y la implantación de las herramientas case}

Hay una variedad de aspectos que deben ser considerados al momento de evaluar la implantación de las herramientas CASE en una determinada empresa. Pareciera evidente que una serie de elementos o factores presentes en el contexto organizacional tienen alguna influencia en el éxito o fracaso de este proceso de cambio. Elementos como la compatibilidad de la metodología de la empresa para el desarrollo de sistemas con las herramientas CASE, el apoyo tanto de la gerencia senior como de la alta gerencia, el nivel de involucramiento del usuario, la estrategia de implementación de los sistemas y el reajuste organizacional deben ser tomados en cuenta cuando se evalúa este tipo de proceso, independientemente de la empresa que se esté considerando [Friesen y Orlikowski (1989), Meyer y Goes (1988), Premkumar y Potter (1995)].

\subsection{Compatibilidad de la metodología con las herramientas CASE}

Es importante examinar si la metodologia que está en uso en el departamento de Sistemas de Información (S.I.) es compatible con la que sugiere o usa la herramienta CASE (Friesen y Orlikowski, 1989). La metodología es crítica en el proceso de innovación de las herramientas CASE, ya que es necesario mediar entre la diferencia del esfuerzo del proceso de desarrollo de sistemas y las herramientas automatizadas que facilitan la ejecución de las tareas (Meyer y Goes, 1988). Según los resultados arrojados en la investigación de estos autores, las organizaciones que asimilaron el proceso de adopción de las herramientas CASE poseían un modelo de desarrollo de sistemas antes de la adquisición de las herramientas.

Por otra parte, según señala Janus, citado en Rowe (1993), "la clave de éxito de alguna herramienta CASE en la organización, es la estandarización de la metodología en el ambiente de desarrollo". Sin embargo, del estudio realizado por Rowe, señala que no existe relación entre la selección y aceptación de la herramienta CASE con la metodología adoptada. Por el contrario, Sumner (1993) plantea que un factor importante para la implementación exitosa de las herramientas CASE es la adherencia a la metodologia seleccionada; y para Jankowski (1995) el soporte para la metodología de desarrollo particular, es uno de los criterios más importantes para la selección de las herramientas CASE.

Del análisis de los datos descriptivos se obtuvo que $46 \%$ de los gerentes señalaron que la metodología utilizada en su organización es propia (creada en casa), un $27 \%$ manifestó que utilizan las herramientas recomendada por los vendedores, un $19 \%$ de los expertos señalaron que la metodología es adaptada de los libros y un $8 \%$ ( 3 gerentes) que cada analista posee su propia metodología. 
En cuanto al análisis inferencial, este item fue operacionalizado a través del reactivo: La disposición a los cambios metodológicos por parte del personal de sistemas de información beneficia la adopción de las herramientas CASE. En este sentido, existió una fuerte tendencia por parte de los gerentes a responder la Categoría De acuerdo, pues las medidas de tendencia central como la mediana y moda están muy cercanas al equivalente numérico de esa categoría que es 4 . Por lo que se puede concluir que se requiere compatibilidad entre la metodologia en uso en el departamento de S.I. con la del CASE lo cual ayuda a la adopción de las herramientas.

Es importante resaltar que un porcentaje importante de empresas no poseen metodología de desarrollo de sistemas, y considerando los niveles de Humphrey, estarían ubicadas en el nivel 1 de madurez del Software Engineering Institute (SEI), (Yourdon, 1993).

En las organizaciones venezolanas estudiadas, la metodología predominante es hecha en casa (propia), por lo que no necesariamente es compatible con las que recomiendan los vendedores de las herramientas. Esto pudiera, como lo señalan Meyery Goes (1988), limitar el éxito de las CASE en las organizaciones.

\subsection{Apoyo por parte de la gerencia senior}

En las compañias involucradas en el estudio realizado por Meyer y Goes (1988), este factor aparece constantemente como facilitador en el proceso de asimilación de las herramientas CASE.
En tal sentido, tener apoyo por parte de la gerencia es un "facilitador importante en el proceso, ya que permite la asimilación a través del esfuerzo motivacional, financiero y de recursos" (Friesen y Orlikowski, 1989: 27), de las herramientas CASE. Si el gerente senior no está involucrado los resultados pueden ser inciertos.

En cuanto al análisis inferencial, éste fue operacionalizado a través de los siguientes reactivos: Para implementar con éxito las herramientas CASE se debe contar con un patrocinador ejecutivo; en el momento de implementar las herramientas CASE se contó con un patrocinador ejecutivo quien hizo el enlace con el negocio; para implementar con éxito las herramientas CASE, se debe contar con un patrocinador operativo con un fuerte perfil de liderazgo; en el momento de implementar las herramientas CASE se contó con un patrocinador operativo con un fuerte perfil de liderazgo. Hubo una marcada tendencia por parte de los gerentes a responder la Categoría De acuerdo a los ítems relacionados con esta variable, pues las medidas de tendencia central como la mediana y la moda fueron el equivalente numérico de la Categoria que es 4 . Por lo que se puede concluir que, existe la percepción de que si se cuenta con el apoyo por parte de la gerencia senior, se facilita la adopción de las CASE.

\subsection{Apoyo por parte de la alta gerencia}

Premkumar y Potter (1995), en su investigación señalan que existe una variable muy importante que diferencia las 
del análisis de la opinión de los gerentes respecto a las variables que surgen de la interacción entre la tecnología y el contexto organizacional que afectan la adopción de las herramientas CASE.

\section{El contexto organizacional y la implantación de las herramientas case}

Hay una variedad de aspectos que deben ser considerados al momento de evaluar la implantación de las herramientas CASE en una determinada empresa. Pareciera evidente que una serie de elementos o factores presentes en el contexto organizacional tienen alguna influencia en el éxito o fracaso de este proceso de cambio. Elementos como la compatibilidad de la metodología de la empresa para el desarrollo de sistemas con las herramientas CASE, el apoyo tanto de la gerencia senior como de la alta gerencia, el nivel de involucramiento del usuario, la estrategia de implementación de los sistemas y el reajuste organizacional deben ser tomados en cuenta cuando se evalúa este tipo de proceso, independientemente de la empresa que se esté considerando [Friesen y Orlikowski (1989), Meyer y Goes (1988), Premkumar y Potter (1995)].

\subsection{Compatibilidad de la metodología con las herramientas CASE}

Es importante examinar si la metodología que está en uso en el departamento de Sistemas de Información (S.I.) es compatible con la que sugiere o usa la herramienta CASE (Friesen y Orlikowski, 1989). La metodología es crítica en el proceso de innovación de las herramientas CASE, ya que es necesario mediar entre la diferencia del esfuerzo del proceso de desarrollo de sistemas y las herramientas automatizadas que facilitan la ejecución de las tareas (Meyer y Goes, 1988). Según los resultados arrojados en la investigación de estos autores, las organizaciones que asimilaron el proceso de adopción de las herramientas CASE poseían un modelo de desarrollo de sistemas antes de la adquisición de las herramientas.

Por otra parte, según señala Janus, citado en Rowe (1993), "la clave de éxito de alguna herramienta CASE en la organización, es la estandarización de la metodología en el ambiente de desarrollo". Sin embargo, del estudio realizado por Rowe, señala que no existe relación entre la selección y aceptación de la herramienta CASE con la metodología adoptada. Por el contrario, Sumner (1993) plantea que un factor importante para la implementación exitosa de las herramientas CASE es la adherencia a la metodologia seleccionada; y para Jankowski (1995) el soporte para la metodología de desarrollo particular, es uno de los criterios más importantes para la selección de las herramientas CASE.

Del análisis de los datos descriptivos se obtuvo que $46 \%$ de los gerentes señalaron que la metodología utilizada en su organización es propia (creada en casa), un $27 \%$ manifestó que utilizan las herramientas recomendada por los vendedores, un $19 \%$ de los expertos señalaron que la metodología es adaptada de los libros y un $8 \%$ ( 3 gerentes) que cada analista posee su propia metodología. 
En cuanto al análisis inferencial, este ítem fue operacionalizado a través del reactivo: La disposición a los cambios metodológicos por parte del personal de sistemas de información beneficia la adopción de las herramientas CASE. En este sentido, existió una fuerte tendencia por parte de los gerentes a responder la Categoría De acuerdo, pues las medidas de tendencia central como la mediana y moda están muy cercanas al equivalente numérico de esa categoría que es 4 . Por lo que se puede concluir que se requiere compatibilidad entre la metodología en uso en el departamento de S.I. con la del CASE lo cual ayuda a la adopción de las herramientas.

Es importante resaltar que un porcentaje importante de empresas no poseen metodología de desarrollo de sistemas, y considerando los niveles de Humphrey, estarian ubicadas en el nivel 1 de madurez del Software Engineering Institute (SEI), (Yourdon, 1993).

En las organizaciones venezolanas estudiadas, la metodología predominante es hecha en casa (propia), por lo que no necesariamente es compatible con las que recomiendan los vendedores de las herramientas. Esto pudiera, como lo señalan Meyer y Goes (1988), limitar el éxito de las CASE en las organizaciones.

\subsection{Apoyo por parte de la gerencia senior}

En las compañías involucradas en el estudio realizado por Meyer y Goes (1988), este factor aparece constantemente como facilitador en el proceso de asimilación de las herramientas CASE.
En tal sentido, tener apoyo por parte de la gerencia es un "facilitador importante en el proceso, ya que permite la asimilación a través del esfuerzo motivacional, financiero y de recursos" (Friesen y Orlikowski, 1989: 27), de las herramientas CASE. Si el gerente senior no está involucrado los resultados pueden ser inciertos.

En cuanto al análisis inferencial, éste fue operacionalizado a través de los siguientes reactivos: Para implementar con éxito las herramientas CASE se debe contar con un patrocinador ejecutivo; en el momento de implementar las herramientas CASE se contó con un patrocinador ejecutivo quien hizo el enlace con el negocio; para implementar con éxito las herramientas CASE, se debe contar con un patrocinador operativo con un fuerte perfil de liderazgo; en el momento de implementar las herramientas CASE se contó con un patrocinador operativo con un fuerte perfil de liderazgo. Hubo una marcada tendencia por parte de los gerentes a responder la Categoría De acuerdo a los ítems relacionados con esta variable, pues las medidas de tendencia central como la mediana y la moda fueron el equivalente numérico de la Categoría que es 4 . Por lo que se puede concluir que, existe la percepción de que si se cuenta con el apoyo por parte de la gerencia senior, se facilita la adopción de las CASE.

\subsection{Apoyo por parte de la alta gerencia}

Premkumar y Potter (1995), en su investigación señalan que existe una variable muy importante que diferencia las 
organizaciones que adoptan las CASE de las que no lo hacen, tal como poseer un promotor interno del producto adquirido. Este vendría a ser una especie de líder que facilitaría la adopción de la herramienta CASE en la empresa. Además, cita a Sumner (1993) quien dice que contar con el apoyo de la alta gerencia es uno de los dos factores importantes para implementar con éxito las herramientas CASE. El apoyo de la gerencia ayuda a que la tecnología sea aceptada, explicando sus beneficios y las consecuencias positivas que traerá después de la implementación de las HC. De esta manera se pueden "reducir las inhibiciones de los empleados y sus temores injustificados" (Premkumar y Potter, 1995: 117).

Es de hacer notar que $76 \%$ de los expertos manifestaron que la adquisición de las herramientas CASE fue liderada por la Gerencia Funcional de Informática, otro $14 \%$ señaló a la alta gerencia.

En relación al análisis inferencial, esta variable (apoyo de la alta gerencia), fue operacionalizada a través del siguiente reactivo: La alta gerencia debe apoyar a los gerentes medios para la incorporación de las herramientas CASE; los gerentes tendieron a responder las categorías 4 y 5 (De acuerdo y Totalmente de acuerdo respectivamente) lo cual es indicador de consenso, pues el porcentaje de respuestas ubicadas en estas categorías supera el $90 \%$. Además las medidas de tendencia central en la mayoría de las preguntas tienden a la Categoría De acuerdo, ya que los valores de la mediana por preguntas es 4. Se infiere que contar con el apoyo por parte de la Alta Gerencia es un fac- tor que influye favorablemente en la adopción de la misma.

\subsection{Involucrar al usuario}

En las investigaciones realizadas por Meyer y Goes (1988: 17), el rol de los usuarios en todas las compañías resultó muy importante como facilitador del proceso de asimilación de las herramientas CASE; ya que "involucrar al usuario aumenta la asimilación de las herramientas CASE debido a las presiones que éstos ejercen sobre los departamentos de S.I. para lograr un mayor uso de ella". Si el usuario final del sistema se siente confiado en que la herramienta le producirá un mejor producto, se inclinará a promover su uso como esquema de desarrollo.

Del análisis descriptivo realizado el $44 \%$ de los gerentes opinaron que los usuarios deben involucrarse en el control de cada fase del desarrollo de los sistemas, un $32 \%$ manifestó que deben ser gerentes de proyecto y un $22 \%$ que los usuarios deben sólo involucrarse en la fase de análisis.

Una proporción importante de gerentes señaló que el usuario debe estar involucrado en el control de cada fase del desarrollo de los sistemas, ya que estar inmerso desde el principio hasta el final del proceso, garantiza el desarrollo y enfoque del sistema hacia las necesidades del negocio, tal como fue señalado por Meyer y Goes (1988).

\subsection{Estrategia de implementación}

La presencia de una estrategia explícita de implementación aparece como 
un factor que facilita la difusión de las herramientas CASE a través de las operaciones de desarrollo de sistemas. Es un aspecto dominante en el proceso seguido por las compañias en la asimilación de la innovación CASE. Meyer y Goes (1988), de la investigación realizada en $10 \mathrm{com}$ pañias identificaron tres estrategias llamadas fases: a) implementación vertical, la cual permite aplicar las herramientas CASE incrementalmente, vía proyectos individuales; es decir, permite realizar un proyecto en todo el ciclo de vida de desarrollo de sistemas; ésta es la que más prevalece, fue usada en 8 de las 10 compañias estudiadas, ya que posee una gran ventaja, las empresas pueden explorar la curva de aprendizaje realizando ajustes en sus metodologías y desarrollando estándares y procedimientos como resultado del aprendizaje de la lección de los proyectos haciendo uso de la CASE; $\mathbf{b}$ ) implementación horizontal, es la que permite tomar los proyectos y para cada uno de ellos se va incrementando, estado por estado, el ciclo de desarrollo, y c) implementación combinada, permite difundir las herramientas CASE en dos direcciones: incrementando proyecto por proyecto como en la opción vertical y, lateralmente, estado por estado como en la alternativa horizontal.

Del análisis de los datos se puede destacar que las estrategias más utilizadas por los gerentes para incorporar la tecnología fueron por igual la vertical y la combinada, con un $35 \%$. El estudio realizado por Meyer y Goes (1988), señala que en EEUU la fase estratégica de implementación vertical fue la que prevale- ció, permitiendo la adopción de las CASE.

Por otra parte, la estrategia más señalada por los gerentes venezolanos para implementar exitosamente la CASE, fue la de involucrar a los usuarios en la implementación de las herramientas CASE con un $31 \%$ de consenso de los expertos, seguida por un $23 \%$ para la estrategia de implementación combinada y un $23 \%$ para el plan con objetivos del negocio.

Resulta evidente que en algunas empresas existe la tendencia de involucrar a los usuarios en la implementación de las CASE, lo cual como lo señalan Kendall y Kendall (1997) "resulta crítico para el desarrollo exitoso de los sistemas", como ventaja adicional encontramos que los clientes cuando están involucrados en todo el desarrollo del sistema pueden solicitar cambios o correcciones sobre la marcha.

\subsection{Reajuste organizacional}

Tal como lo refiere Alvin Toffler, citado por Faria (1992), la empresa tiene que adaptarse a cambios internos y externos, y para hacerlo tiene que introducir cambios estructurales, llevando a muchos individuos a ocupar nuevos cargos, recibir nuevas responsabilidades, pasar sus antiguas responsabilidades a otras personas. El individuo necesita una nueva actitud psicológica y emocional, para poder adaptarse a los cambios en la organización.

Las empresas que tienen un grupo para administrar las herramientas CASE, $\mathrm{HC}$, se caracterizan por ser organizaciones complejas con grandes organigra- 
mas, con muchos empleados y un alto nivel de madurez en informática (Viloria, 1995).

Las empresas que carecen de este grupo se clasifican en: las que poseen líderes de proyectos expertos en $\mathrm{HC}$ y las que no; en éstas últimas, los analistas deben aprender a utilizarlas por sí solos 0 con alguna ayuda técnica. Estas organizaciones se encuentran en los niveles más bajos del modelo del Instituto de Ingeniería de Software, SEI.

Para evaluar el impacto de los grupos internos en el proceso de adopción de las herramientas CASE, se agruparon las empresas en ocho (8) categorías con la finalidad de determinar diferencias al respecto dependiendo del tipo de organización, en la Tabla 1 se pueden observar las estadísticas relacionadas con la opinión de los gerentes en cuanto a la crea- ción de grupos de soporte antes y después de la incorporación de las herramientas CASE.

En cuanto a la creación de grupos antes de la incorporación de las CASE, el $67 \%$ de los gerentes de la industria petrolera manifestaron haber creado grupos formales para darles soporte a los analistas que usarian las herramientas CASE. Todos los gerentes de las empresas de seguros señalaron que se crearon grupos formales para el soporte de las CASE y, que como consecuencia, hubo cambios en su estructura organizacional. Sólo un $25 \%$ de los gerentes correspondientes a la banca comercial indicaron que se creó un grupo formal. Según Kwok y Arnett (1993), es más probable que aquellas empresas que realizan cambios en su estructura organizativa tengan éxito en la incorporación de las CASE.

Tabla 1

Percepción relacionadas con la creación de grupo interno encargado de administrar o dar apoyo a los analistas antes (A) y después (D) de la incorporación de las herramientas CASE

\begin{tabular}{|c|c|c|c|c|c|c|c|c|}
\hline \multirow[t]{2}{*}{ Tipo de Empresas } & \multirow[t]{2}{*}{ Cantidad } & \multicolumn{2}{|c|}{$\begin{array}{c}\text { Grupo Formal } \\
(\%)\end{array}$} & \multicolumn{2}{|c|}{$\begin{array}{c}\text { Grupo Informal } \\
(\%) \\
\end{array}$} & \multicolumn{2}{|c|}{$\begin{array}{c}\% \text { No se creó } \\
\text { grupo }\end{array}$} & \multirow[t]{2}{*}{ Total } \\
\hline & & A & $\mathrm{D}$ & $A$ & $D$ & $A$ & $\mathrm{D}$ & \\
\hline Petrolera & 5 & 67 & 67 & 22 & 22 & 11 & 11 & $100 \%$ \\
\hline $\begin{array}{l}\text { Desarrolladores/ vendedores } \\
\text { de software/Consultores }\end{array}$ & 16 & 7 & 0 & 57 & 57 & 36 & 43 & $100 \%$ \\
\hline Universidades & 1 & 0 & 0 & 0 & 0 & 100 & 100 & $100 \%$ \\
\hline Banca Comercial & 5 & 25 & 50 & 25 & 0 & 50 & 50 & $100 \%$ \\
\hline Empresas de Seguros & 2 & 100 & 100 & 0 & 0 & 0 & 0 & $100 \%$ \\
\hline Alimentos y Bebidas & 4 & 0 & 33 & 50 & 33 & 50 & 17 & $100 \%$ \\
\hline Electricidad, Gas y Agua & 1 & 0 & 0 & 100 & 100 & 0 & 0 & $100 \%$ \\
\hline $\begin{array}{l}\text { Transporte, Almacenamiento } \\
\text { y Comunicaciones }\end{array}$ & 1 & 0 & 0 & 100 & 100 & 0 & 0 & $100 \%$ \\
\hline
\end{tabular}

Fuente: Tomado De Freitas (1997). 
Por otra parte, en cuanto a la creación de grupos después de la incorporación de las $\mathrm{HC}$, el $38 \%$ de los expertos señalaron que se creó un grupo informal en la organización para darle soporte a los analistas después de haberse incorporados las herramientas CASE. Un $29 \%$ de los gerentes indicaron que se creó un grupo formal en la organización y otro $29 \%$ manifestó no haberse creado ningún grupo. De nuevo, es de esperar que aquellas empresas que crearon grupos de soporte a sus analistas tengan más éxito en el proceso de incorporación de las herramientas CASE.

Esta variable fue operacionalizada a través de los siguientes reactivos: En el momento de implementar las $\mathrm{HC}$, se debe crear un grupo interno encargado de administrar o dar apoyo a los analistas que la utilicen; cuando se incorporan las $\mathrm{HC}$ debe haber cambios en la estructura organizativa del Departamento de S.I. Existe un nivel de consenso hacia el rechazo del enunciado de la pregunta, ya que más del $50 \%$ de los gerentes entrevistados están ubicados en las Categorías 1 y 2 correspondientes a Totalmente en desacuerdo y En desacuerdo respectivamente, y un $27 \%$ están ubicados en la Categoría de Indeciso. Por otra parte, la tendencia fue a responder En desacuerdo, pues su mediana y su moda tienen un valor igual a 2 . Se infiere que la existencia o no del reajuste organizacional no influye en la adopción de las CASE.

Las organizaciones que han alcanzado un mayor nivel de madurez en el uso de la tecnología de la información sufren cambios en la estructura organizacional como consecuencia de la implementación de las herramientas CASE.

La industria petrolera posee grupos que administran las herramientas CASE, la mitad de las empresas de la banca comercial poseen grupo de soporte para las CASE y las empresas de seguros también tienen grupos formales de soporte.

Es importante resaltar que las organizaciones que crearon estos grupos son aquellas que poseían un mayor nivel de madurez en informática, cumpliéndose lo señalado por Leavitt, citado por Kwok y Arnett (1993), que la CASE ocasiona reajuste organizacionales.

Por lo tanto, el crear grupos formales que les sirvan de apoyo a los analistas, es una variable que influye favorablemente en la adopción de las herramientas CASE.

\section{Conclusiones}

De las investigación realizada destacan las siguientes conclusiones:

a. Contar con una metodología compatibie con la asociada a la herramienta CASE facilita su incorporación, tal como fue señalado por Friesen y Orlikowski (1989).

b. El apoyo de la gerencia senior es considerado por los gerentes venezolanos como variable que facilita el proceso de asimilación de las CASE, como fue señalado por Meyer y Goes (1988).

c. Contar con el apoyo de la alta gerencia, es considerado por los gerentes venezolanos como una variable que facilita el proceso de adopción de las CASE, de esta forma se corrobora la 
conclusión de Premkumar y Potter (1995) en el caso venezolano.

d. Involucrar al usuario en el control de cada fase del desarrollo de sistemas facilita la incorporación.

e. Contar con una estrategia de implementación de las CASE facilita su adopción.

\section{Bibliografía citada}

Burch, J. y Grudnitski, G. (1992). Diseño de Sistemas de Información. 1ra edic. México: Edit Grupo Noriega $E$.

De Freitas, $V$. (1997). Factores que Inciden en la Adopción de las Herramientas CASE en las Organizaciones venezolanas. Tesis de Grado en Ingeniería de Sistemas. Mimeo. Universidad Simón Bolívar. Caracas. Venezuela.

De Freitas, V. (1998). Factores Organizacionales que Inciden en la Adopción de las Herramientas CASE en las Organizaciones venezolanas. II Congreso Iberoamericano de Psicología. Julio. Madrid. España.

De Freitas, V. (1999). "Factores Tecnológicos que Inciden en la Adopción de las Herramientas CASE en las Organizaciones venezolanas". Revista Venezolana de Gestión Tecnológica. Vol. 20. $N^{\circ} 2, \mathrm{pp} .43-58$.

Electronic Computer Glossary. Computer Select, may 1996.

Faria, F. (1992). Desarrollo Organizacional. Enfoque Integral. México: Editorial Limusa.

Friesen, M. y Orlikowski, W. (1989). Assimilating CASE Tools in Organizations. Center for Information Systems research, Sloan School of Management, October.
Jankowski, D. (1995). "CASE Tool Selection. Using Methodology Support to Choose the Right Tool for the job". Journal of Systems Management. July/August, v.46. pp20-26.

Kemerer, C. (1992). "Learning Curve Models For Integrated CASE tool Management". Mit Center for Information System Research. IEEE Software.

Kendall, K. y Kendall, J. (1997). Análisis y Diseño de Sistemas. 3ra edic. México: Prentice Hall Hispanoamericana, S.A.

Kwok, L.K.L. y Arnett, K.P. (1993). “Organizational Impact of CASE Technology". Journal of Systems Management. v44.

Meyer, A. y Goes, J. (1988). "Organizational Assimilation of Innovations: A Multilevel Contextual Analysis". Academy of Management Journal. 31(4), pp 897-923.

Premkumar, G. y Potter, M. (1995). "Adoption of Computer Aided Software Engineering (CASE) Technology: An Innovation Adoption Perspective". Data Base Advances. May/August, v26. pp 105-123.

Rowe, J. (1993). "Can Enforced Standardization Affect Case Usage?". Journal of Systems Management. march, v44. pp 29-33.

Sumner, M. (1993). "Factors Influencing the Adoption of CASE". In Thomas J. Bergin (Ed.), Computer-Aided Software Engineering. Harrisburg, PA: Idea Group Publishing, pp 130-155.

Viloria, O. (1995). Efectos de las Herramientas CASE sobre las Organizaciones Venezolanas. Tesis de Grado en Ingeniería de Sistemas. Mimeo. Universidad Simón Bolivar. Caracas. Venezuela.

Yourdon, E. (1993). Decline \& Fall of the American Programmer. Yourdon Press. Cap4, pp 73-75. 